\title{
Indels within the bovine visfatin gene affect its mRNA expression in longissimus muscle and subcutaneous fat
}

\author{
H. Cai, Z. Wang, X. Lan, Y. Xu, H. Chen, and C. Lei \\ College of Animal Science and Technology, Northwest A\&F University, Yangling, Shaanxi, 712100, China \\ Correspondence to: H. Chen (chenhong1212@263.net)
}

Received: 24 September 2015 - Revised: 6 January 2016 - Accepted: 18 January 2016 - Published: 17 February 2016

\begin{abstract}
Visfatin, an adipokine hormone produced primarily by visceral adipose tissue in mammals, has been identified as having a crucial role in growth and development of skeletal muscle and lipids. In this research, the effects of two indel loci (35 bp indel: AC_000161.1: g. 20540-20541 Ins ACTGGAATTCTAGTTTAAAAATTGCTACTAATGAA located in intron 4; 6 bp indel: AC_000161.1: g. 25873-25878 Del: TAAAAA located in intron 5) of the visfatin gene on mRNA expression levels were studied by means of real-time quantitative PCR (qPCR) in longissimus muscle and subcutaneous fat from 95 Qinchuan cattle. Firstly, visfatin expression level in longissimus muscle of fetal cattle was prominently greater than that in calves and adult cattle $(P<0.05)$. The expression level of visfatin in subcutaneous fat was notably higher than that in longissimus muscle of calves and adult cattle $(P<0.05)$. Secondly, there were three genotypes (ins/ins, del/del and ins/del) and two genotypes (ins/del and ins/ins) detected in the $35 \mathrm{bp}$ locus and $6 \mathrm{bp}$ locus, respectively. Visfatin showed a minimum expression level in longissimus muscle in the homozygous deletion genotype at the $35 \mathrm{bp}$ indel locus. Especially in calves, expression of visfatin was significantly greater in the heterozygous genotype than that in the homozygous insertion genotpye $(P<0.05)$. No statistical differences were found among visfatin expression level based on genotypes in the $6 \mathrm{bp}$ indel locus $(P>0.05)$. Compared to heterozygous genotype, the expression level of homozygous insertion genotype was lower in longissimus muscle but greater in subcutaneous fat. These results imply that the expression levels of bovine visfatin vary with age and its indels might be putative variants mediating the expression of the bovine visfatin gene. This study provides useful information for further functional studies of bovine visfatin.
\end{abstract}

\section{Introduction}

Visfatin, an adipokine hormone, is secreted and abundantly expressed in visceral adipose tissue in mammals (Steppan et al., 2001). Initially, it was discovered as Nampt (nicotinamide phosphoribosyltransferase), which catalyzes the condensation of nicotinamide with 5-phosphoribosyl-1pyrophosphate to yield nicotinamide mononucleotide, an intermediate in the biosynthesis of NAD (nicotinamide adenine dinucleotide; Magni et al., 1999). Subsequently, it was identified as PBEF (pre-B-cell colony-enhancing factor) induced by pokeweed mitogen and cycloheximide, and the PBEF can strengthen the effect of the stem cell factor and interleukin 7 in pre-B cell colony formation (Samal et al., 1994). Visfatin has been found to be widely expressed in multiple tissues and organs in mammals, including skeletal muscle and adipose tissues (Klöting and Klöting, 2005; McGlothlin et al., 2005; Chen et al., 2007). All of these studies indicated that visfatin was absolutely necessary for the individual growth and development. However, there are very few reports about bovine visfatin, particularly with regard to its role in bovine skeletal muscle and fat.

In the last few years, genetic variants of the visfatin gene have been identified as molecular markers and showed significant associations with normal growth and development of animals and humans (Bailey et al., 2006; Jian et al., 2006; Blakemore et al., 2009). Among them, two indels (35 bp indel: AC_000161.1: g. 20540-20541 Ins ACTGGAATTCTAGTTTAAAAATTGCTACTAATGAA in intron 4; 6 bp indel: AC_000161.1: g. 25873-25878 Del: TAAAAA in intron 5) of the bovine visfatin gene were significantly associated with growth traits in Chinese cattle (Wang et al., 2009, 
Table 1. Specific primers for qPCR.

\begin{tabular}{llcc}
\hline Gene & Primer sequence $\left(5^{\prime}\right.$ to $\left.3^{\prime}\right)$ & Length (bp) & Annealing $\left({ }^{\circ} \mathrm{C}\right)$ \\
\hline Visfatin & F: CTACTCGCTCCCTTTCCC & 160 & 60 \\
& R: GCCGCAGCATTCATCTCG & & \\
GAPDH & F: TGTTTGTGATGGGCGTGAACCA & 154 & 60 \\
& R: ATGGCGTGGACAGTGGTCATAA & & \\
\hline
\end{tabular}

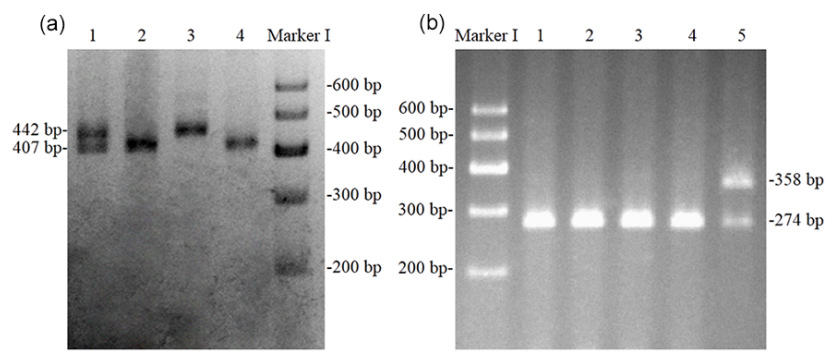

Figure 1. Agarose gel electrophoresis patterns of two indel loci. (a) Agarose gel electrophoresis detecting the genotypes at the $35 \mathrm{bp}$ locus. The band patterns in lane 1, 2, 3 and 4 represent the ins/del, del/del, ins/ins and del/del genotype, respectively. (b) Agarose gel electrophoresis detecting the genotypes at the $6 \mathrm{bp}$ indel locus. The band patterns in lane 1-4 and 5 represent the del/del and ins/del genotype, respectively.

2012). Previous studies have reported that genetic variations, such as SNPs (single-nucleotide polymorphisms), indels (small insertions and deletions), and CNVs (copy number variants), may be involved in mRNA regulation (Hogg and Harries, 2014) and gene expression (Msalya et al., 2010; $\mathrm{Xu}$ et al., 2013), but the mechanisms caused by these mutations within the visfatin gene have not been well clarified.

Herein, given the limited information about bovine visfatin expression level and the operating principle of its sequence variations, the expression levels of the bovine visfatin gene were detected in longissimus muscle and subcutaneous fat at different growth stages. Furthermore, association analysis was performed based on the hypothesis that indels of the visfatin gene were associated with its mRNA level. Thus, the aim of this study was to examine the effect of the 35 and $6 \mathrm{bp}$ indel loci on the expression levels of the visfatin gene in Qinchuan cattle.

\section{Materials and methods}

\subsection{Tissue sample collection, genomic DNA and total RNA isolation, and CDNA synthesis}

Tissue samples, including longissimus muscle and subcutaneous fat, were collected from 61 fetal cattle ( 26 female and 35 male, 90 days of gestation), 10 male calves (newborn) and 24 adult male cattle (24 months old). Samples were frozen in liquid nitrogen as soon as they were separated, then stored at $-80^{\circ} \mathrm{C}$ before use. All cattle were of the Qinchuan breed kept in Shaanxi Province. Genomic DNA from longissimus muscle and subcutaneous fat was extracted by proteinase $K$ digestion, chloroform extraction and absolute ethanol precipitation. Total RNA was isolated using RNAiso Plus (TaKaRa, Japan) according to the instructions. Then cDNA was synthesized using a PrimeScript RT Reagent Kit (TaKaRa, Japan). The quality of DNA and RNA was evaluated by Nandrop 2000 spectrophotometry (Thermo, USA).

\subsection{Genotyping}

In order to evaluate differences among expression level based on genotypes at two indel loci, individual genotypes should be detected firstly following Wang et al. (2009, 2012). In detail, the genotypes in the $35 \mathrm{bp}$ indel locus were detected directly by means of electrophoresis of PCR products with $2.5 \%$ agarose gel (Fig. 1a). At the $6 \mathrm{bp}$ indel locus, the PCR products were digested with Dra I endonuclease, and the digested fragments were detected in $2.5 \%$ agarose gel (Fig. 1b).

\subsection{Quantitative PCR (qPCR)}

Gene-specific primers of the bovine visfatin gene and a housekeeping gene, glyceraldehyde-3-phosphate dehydrogenase $(\mathrm{GAPDH})$, chosen as a reference gene, were designed using Primer 5.0 (Table 1) and verified with Primer-BLAST (http://www.ncbi.nlm.nih.gov/tools/primer-blast). qPCR was carried out in triplicate with SYBR premix ExTaq II (TaKaRa, Japan) and a CFX 96TM real-time detection system (BioRad, USA) according to the manufacturer's recommendations.

\subsection{Statistical analysis}

The relative expression ratios were calculated as $2^{-\Delta \Delta \mathrm{Ct}}$ following the Schmittgen and Livak method (2008). The data were shown as mean $\pm \mathrm{SE}$ (standard error). Independent $t$ tests were performed to support the visfatin gene expression of the normally distributed data following the general linear model below:

$Y=\mu+G_{i}+A_{j}+S_{k}+G_{i} A_{j}+G_{i} S_{k}+A_{j} S_{k}+G_{i} A_{j} S_{k}+E_{i j k}$,

where $Y$ is expression ratio, $\mu$ the overall mean, $G_{i}$ the effect of the $i$ th genotype, $A_{j}$ the effect of the $j$ th age, $S_{k}$ the effect 
Table 2. Individual numbers for every genotype at two indel loci. The values in the brackets are the genotypic frequencies. A dash means that the genotype was not detected.

\begin{tabular}{lcccccc}
\hline \multirow{2}{*}{ Age } & \multirow{2}{*}{ Total } & \multicolumn{3}{c}{35 bp indel locus } & \multicolumn{2}{c}{6 bp indel locus } \\
\cline { 3 - 7 } & & Ins/ins & Ins/del & Del/del & Ins/ins & Ins/del \\
\hline Fetal cattle & 61 & 4 & 12 & 45 & 59 & 2 \\
& & $1(0.066)$ & $(0.197)$ & $(0.738)$ & $(0.967)$ & $(0.032)$ \\
Calf & 10 & $2_{-}$ & 3 & 7 & 10 & - \\
& & & $(0.300)$ & $(0.700)$ & $(1.000)$ & - \\
Adult cattle & 24 & 2 & 10 & 12 & 22 & 2 \\
& & $(0.083)$ & $(0.417)$ & $(0.500)$ & $(0.917)$ & $(0.083)$ \\
\hline
\end{tabular}

of the $k$ th sex, and $E_{i j k}$ random error (Msalya et al., 2011). $P<0.05$ was considered significant in this research.

\section{Results}

\subsection{DNA genotyping analysis}

The genomic DNA from a total of 95 Qinchuan cattle, including 61 fetal cattle, 10 calves and 24 adult cattle, was genotyped for polymorphisms in the 35 and $6 \mathrm{bp}$ indel loci of the bovine visfatin gene. Three genotypes were found in the $35 \mathrm{bp}$ indel site and two were found in the $6 \mathrm{bp}$ indel site. The individual numbers of every genotype at each locus are shown in Table 2. Compared to the insertion allele, the frequency of the deletion allele was higher in the $35 \mathrm{bp}$ site but lower in the $6 \mathrm{bp}$ site, which is in agreement with previous reports (Wang et al., 2009, 2012).

\subsection{Visfatin gene expression related to ages in longissimus muscle and subcutaneous fat}

In order to investigate the effect of age on visfatin expression, mRNA levels in longissimus muscle and subcutaneous fat among three growth stages were compared using $t$ tests. As shown in Fig. 2, the visfatin expression level in longissimus muscle of fetal cattle was significantly higher than that in both calves $(P=0.000)$ and adult cattle $(P=0.000)$. Adult bovines presented a slightly greater value of mRNA level than calves. In subcutaneous fat, adult cattle had a lower expression level of visfatin mRNA than calves $(P>0.05)$. On the other hand, the level in subcutaneous fat of fetal cattle was significantly greater than that in longissimus muscle of both calves $(P=0.000)$ and adult cattle $(P=0.002)$. Therefore, age notably influenced the visfatin expression, and the expression quantity of visfatin in fat was significantly higher than that in skeletal muscle.

\subsection{Gene expression of visfatin with different genotypes in longissimus muscle and subcutaneous fat}

As can be clearly seen in Fig. 3a, the expression of the visfatin gene in longissimus muscle among genotypes at the

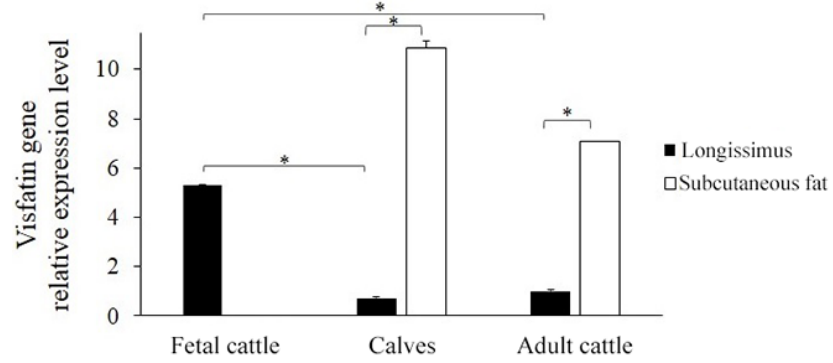

Figure 2. Expression of the visfatin gene (mean \pm SE) related to ages in longissimus muscle and subcutaneous fat. An asterisk means significance difference in the visfatin expression $(P<0.05)$.

$35 \mathrm{bp}$ indel locus suggests the same order in three different growth stages: ins/ins > ins/del > del/del (ins/ins genotype was not detected in calves). In particular, the heterozygous genotype of claves had higher visfatin mRNA than the homozygous deletion genotype $(P=0.048)$. However, there was no statistical significance among these genotypes in fetal and adult cattle $(P>0.05)$. For subcutaneous fat, the visfatin gene displayed different expression levels with no significance among these genotypes $(P>0.05)$. However, the individuals with the ins/del genotype had lower expression than those with del/del genotypes in both calves and adult cattle (Fig. 3b).

On the other hand, at the $6 \mathrm{bp}$ indel locus, statistical significance was not found in either longissimus muscle or subcutaneous fat among visfatin expression based on genotypes $(P>0.05)$. However, the groups with the ins/del genotype showed greater visfatin level than those with the ins/ins genotype in both fetal and adult cattle (Fig. 3c). However, the visfatin expression level demonstrated an order of ins/ins > ins/del in subcutaneous fat in adult cattle (Fig. 3d). (Only one genotype was found at the 6 bp indel site in calves; thus, genotype-phenotype association analysis was not carried out.)

\section{Discussion}

In the last few years, it has been revealed that visfatin plays an essential part in the development of skeletal muscle and adipose tissue. In order to add to the research on visfatin in cattle, in this study, the expression of the bovine visfatin gene referring to ages and genotypes of two indel loci was evaluated to reveal internal correlation of the bovine visfatin gene and its expression pattern in longissimus muscle and subcutaneous fat.

qPCR was applied to evaluate the expression level of visfatin in longissimus muscle and subcutaneous fat during three different growth stages of Qinchuan cattle. Results showed that the highest peak of visfatin mRNA in skeletal muscle appeared at the fetal stage. Previous studies have suggested that visfatin affected the expression of MRFs (myo- 

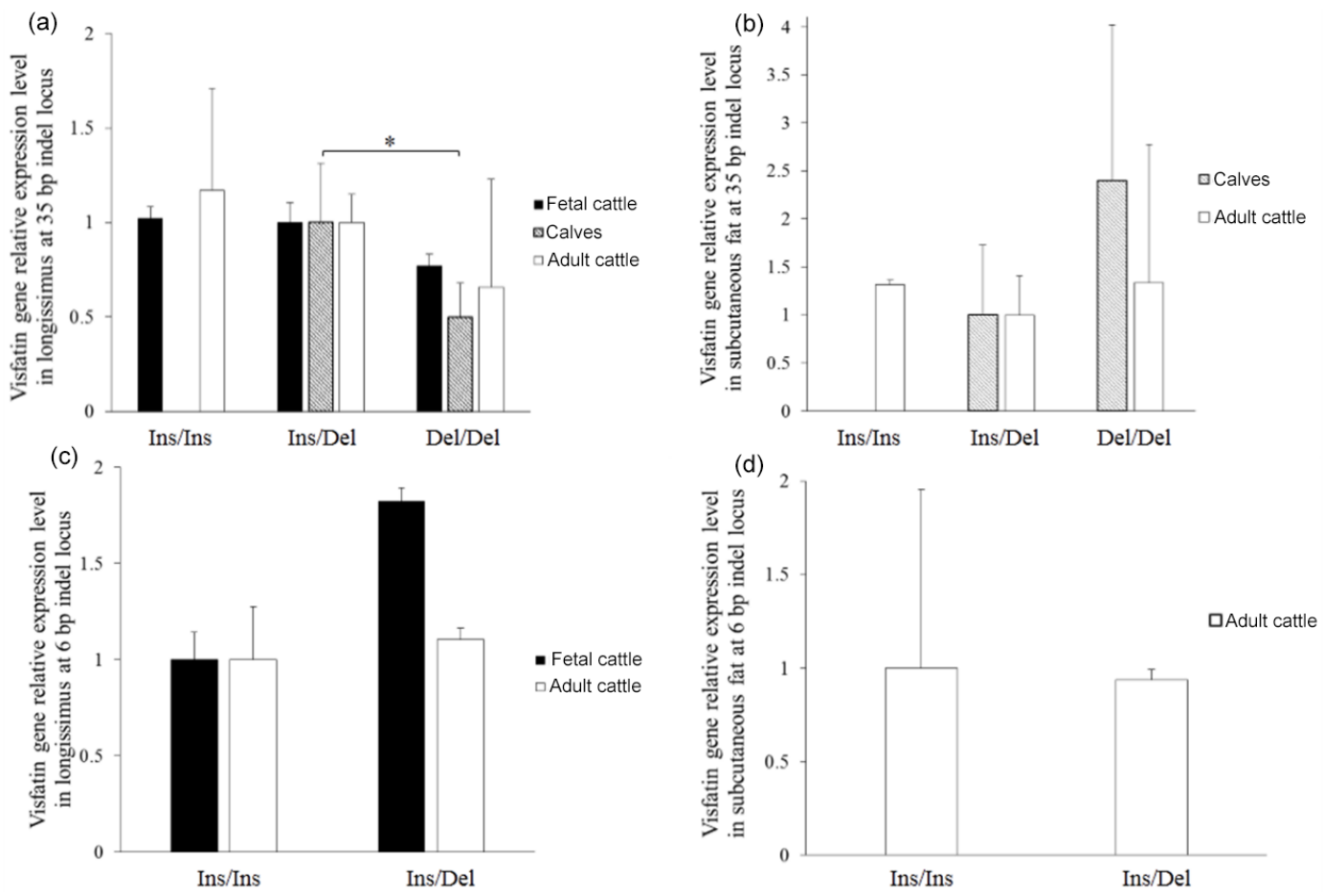

Figure 3. Expression of the visfatin gene (mean $\pm \mathrm{SE}$ ) based on genotypes in two indel loci in longissimus muscle and subcutaneous fat. Panels (a) and (b) present the expression of visfatin in genotypes at the $35 \mathrm{bp}$ indel locus in longissimus muscle and subcutaneous fat, respectively. Panels (c) and (d) present the expression of visfatin related to genotypes at the 6 bp indel locus in longissimus muscle and subcutaneous fat, respectively. An asterisk means significant difference in the visfatin expression $(P<0.05)$. As there is only one genotype detected at the $6 \mathrm{bp}$ indel locus in calves, an association analysis is not performed and its expression level is not shown in the figure.

genic regulatory factors), which are important muscle determination factors during embryonic skeletal muscle development (Braun and Gautel, 2011). Therefore, it was supposed that visfatin might have an important influence on embryonic myogenic programming, which, however, is in need of further functional studies. In addition to the findings, visfatin mRNA was transcribed more in adult cattle than calves in longissimus muscle, which is similar to what is shown in Tang et al. (2000) and Krzysik-Walker et al. (2008), who showed that visfatin level in skeletal muscle increased with age.

All DNA samples of the tested Qinchuan cattle were genotyped at the 35 and $6 \mathrm{bp}$ indel loci of the bovine visfatin gene. Genotypic and allelic types and frequencies agreed with those published in previous reports (Wang et al., 2009, 2012). Next, the effect of these genotypes on mRNA expression was studied. For longissimus muscle, at the $35 \mathrm{bp}$ indel locus, it has been shown that the tendency of visfatin expression was ins/ins $>$ ins/del $>$ del/del in fetal and adult cattle, while the calves with the ins/del genotype had higher expression than those with the del/del genotype. These results suggest that, at the $35 \mathrm{bp}$ indel locus, the cattle with the insertion allele had a higher visfatin level compared to those with the deletion allele. By that analogy, at the $6 \mathrm{bp}$ indel locus, the individuals with the deletion allele had a higher visfatin expression level than those with the insertion allele. Interestingly, growth traits of Qinchuan cattle, which were significantly associated with these two indel loci, presented a completely inverse order of del/del $>$ ins/del $>$ ins/ins and ins/ins $>$ ins/del based on genotypes at the 35 and $6 \mathrm{bp}$ indel loci, respectively (Wang et al., 2009, 2012). Therefore, it was hypothesized that visfatin might negatively regulate the development of longissimus muscle. Notably, the difference in visfatin level between ins/del and del/del was significant in longissimus muscle of calves at the $35 \mathrm{bp}$ indel locus, which suggested that this locus, a potential molecular marker remarkably related to growth traits, could have a noteworthy influence on growth traits by prominently changing visfatin expression. At the $35 \mathrm{bp}$ indel locus, in all stages, the cattle with the del/del genotype showed the lowest expression level in skeletal muscle, but they showed the lowest highest level in fat. At the $6 \mathrm{bp}$ indel locus, visfatin expression levels in skeletal muscle and fat indicated a reverse trend based on genotpyes. Therefore, it was assumed that the function of visfatin in skeletal muscle and fat was inverse. This suggestion is in agreement with previous studies, which have indicated that visfatin can promote adipocytes differentiation, adipose synthesis and accumulation (Klöting and Klöting, 2005); however, it is associated with inhibition of skeletal myoblast differentiation (Fulco et al., 2008). It was perhaps because of 
visfatin being a regulator of visceral adipose tissue that no statistical significance was discovered among subcutaneous visfatin in fat expression based on genotypes.

In summary, here the effects of indel variations on visfatin expression in longissimus muscle and subcutaneous fat were reported. Results showed that the expression of visfatin in longissimus muscle and subcutaneous fat varied with age, and the $35 \mathrm{bp}$ indel locus might be significantly associated with visfatin expression level in skeletal muscle. However, a larger population and more in-depth functional studies may be helpful for better understanding of bovine visfatin in skeletal muscle and fat development.

Acknowledgements. This study was supported by the National Natural Science Foundation of China (no. 31272408), the Program of National Beef Cattle Industrial Technology System (no. CARS38), and the National 863 Program of China (no. 2013AA102505).

Edited by: S. Maak

Reviewed by: G. Liu and one anonymous referee

\section{References}

Bailey, S. D., Loredo-Osti, J. C., Lepage, P., Faith, J., Fontaine, J., Desbiens, K. M., Hudson, T. J., Bouchard, C., Gaudet, D., Perusse, L., Vohl, M. C., and Engert, J. C.: Common polymorphisms in the promoter of the visfatin gene (PBEF1) influence plasma insulin levels in a French-Canadian population, Diabetes, 55, 2896-2902, 2006.

Blakemore, A. I., Meyre, D., Delplanque, J., Vatin, V., Lecoeur, C., Marre, M., Tichet, J., Balkau, B., Froguel, P., and Walley, A. J.: A rare variant in the visfatin gene (NAMPT/PBEF1) is associated with protection from obesity, Obesity, 17, 1549-1553, 2009.

Braun, T. and Gautel, M.: Transcriptional mechanisms regulating skeletal muscle differentiation, growth and homeostasis, Nat. Rev. Mol. Cell. Bio., 12, 349-361, 2011.

Chen, H., Xia, T., Zhou, L., Chen, X., Gan, L., Yao, W., Peng, Y., and Yang, Z.: Gene organization, alternate splicing and expression pattern of porcine visfatin gene, Domest. Anim. Endocrin., 32, 235-245, 2007.

Fulco, M., Cen, Y., Zhao, P., Hoffman, E. P., McBurney, M. W., Sauve, A. A., and Sartorelli, V.: Glucose restriction inhibits skeletal myoblast differentiation by activating SIRT1 through AMPK-mediated regulation of Nampt, Dev. Cell., 14, 661-673, 2008.

Hogg, D. R. and Harries, L. W.: Human genetic variation and its effect on miRNA biogenesis, activity and function, Biochem. Soc. T., 42, 1184-1189, 2014.

Jian, W. X., Luo, T. H., Gu, Y. Y., Zhang, H. L., Zheng, S., Dai, M., Han, J. F., Zhao, Y., Li, G., and Luo, M.: The visfatin gene is associated with glucose and lipid metabolism in a Chinese population, Diabetic. Med., 23, 967-973, 2006.
Klöting, N. and Klöting, I.: Visfatin: gene expression in isolated adipocytes and sequence analysis in obese WOKW rats compared with lean control rats, Biochem. Bioph. Res. Co., 332, 1070-1072, 2005.

Krzysik-Walker, S. M., Ocon-Grove, O. M., Maddineni, S. R., Hendricks III, G. L., and Ramachandran, R.: Is visfatin an adipokine or myokine? Evidence for greater visfatin expression in skeletal muscle than visceral fat in chickens, Endocrinology, 149, 15431550, 2008.

Magni, G., Amici, A., Emanuelli, M., Raffaelli, N., and Ruggieri, S.: Enzymology of NAD+ synthesis, Adv. Enzymol. RAMB, 73, 135-182, 1999.

McGlothlin, J. R., Gao, L., Lavoie, T., Simon, B. A., Easley, R. B., Ma, S. F., Rumala, B. B., Garcia, J. G., and Ye, S. Q.: Molecular cloning and characterization of canine pre-B-cell colonyenhancing factor, Biochem. Genet., 43, 127-141, 2005.

Msalya, G., Shimogiri, T., Nishitani, K., Okamoto, S., Kawabe, K., Minesawa, M., and Maeda, Y.: Indels within promoter and intron 1 of bovine prion protein gene modulate the gene expression levels in the medulla oblongata of two Japanese cattle breeds, Anim. Genet., 41, 218-221, 2010.

Msalya, G., Shimogiri, T., Ohno, S., Okamoto, S., Kawabe, K., Minezawa, M., and Maeda, Y.: Evaluation of PRNP expression based on genotypes and alleles of two indel loci in the medulla oblongata of Japanese Black and Japanese Brown cattle, PLos. ONE., 6, e18787, doi:10.1371/journal.pone.0018787, 2011.

Samal, B., Sun, Y., Stearns, G., Xie, C., Suggs, S., and McNiece, I.: Cloning and characterization of the cDNA encoding a novel human pre-B-cell colony-enhancing factor, Mol. Cell. Biol., 14, 1431-1437, 1994.

Schmittgen, T. D. and Livak, K. J.: Analyzing real-time PCR data by the comparative CT method, Nat. Protoc., 3, 1101-1108, 2008.

Steppan, C. M., Bailey, S. T., Bhat, S., Brown, E. J., Banerjee, R. R., Wright, C. M., Patel, H. R., Anima, R. S., and Lazar, M. A.: The hormone resistin links obesity to diabetes, Nature, 409, 307-312, 2001.

Tang, H., Cheung, W. M., Ip, F. C., and Ip, N. Y... Identification and characterization of differentially expressed genes in denervated muscle, Mol. Cell. Neurosci., 16, 127-140, 2000.

Wang, M., Yu, H., Chen, H., Lan, X. Y., Zhang, L. Z., Zhao, M., Lai, X. S., Wang, X. L., Wang, K. Y., and Wang, J. Q.: Novel 35bp insertion in Visfatin Gene in Chinese cattle, Mol. Biol., 43, 557-561, 2009.

Wang, M., Zhang, Y., Yu, H., Lai, X. S., Zhu, J. L., Jiao, J. Z., Lan, X. Y., Lei, C. Z., Zhang, L. Z., and Chen, H.: Novel 6-bp Deletion Mutation in visfatin Gene and Its Associations with Birth Weight and Bodyweight in Chinese Cattle, J. Integr. Agr., 11, 1327-1332, 2012.

Xu, Y., Zhang, L., Shi, T., Zhou, Y., Cai, H., Lan, X., Zhang, C., Lei, C., and Chen, H.: Copy number variations of MICAL-L2 shaping gene expression contribute to different phenotype of cattle, Mamm. Genome., 24, 208-516, 2013. 